\title{
Almost periodic functions on groupoids
}

\author{
FARID BEHROUZI (*)
}

ABSTRACT - In this paper we generalize the notion of almost periodic functions on groups to the corresponding notion for groupoids. We prove a number of theorems about almost periodic functions in this general setting. We show that the set of almost periodic functions on a groupoid $G, A P(G)$, is a $\mathrm{C}^{*}$-subalgebra of $\ell^{\infty}(G)$. We investigate some topological properties of the maximal ideal space of $A P(G), \mathfrak{b}(G)$, and we obtain a continuous partial operation on $\mathfrak{b} G$. Also, we study almost periodic functions on groupoids defined by an equivalence relation on a set $X$ and obtain a compactification of $X$.

Mathematics Subject Classification (2010). 22A22.

KEYWORDS. Groupoid, almost periodic function.

\section{Introduction}

For a locally compact space $X$, every unital $\mathrm{C}^{*}$-subalgebra $A$ of $C_{b}(X)$ induces a compactification of $X$. In fact the maximal ideal space of $A$ is a compactification of $X$, see [4]. Here $C_{b}(X)$ is the algebra of bounded continuous complex-valued functions on locally compact space $X$. It is interesting to know that if $X$ has further algebraic structures, which compactifications of $X$ preserve these algebraic structures. For example, if $S$ is a discrete semigroup and $A=\ell^{\infty}(S)=C_{b}(S)$, then the algebra $A$ induces $\beta S$, the Stone-Čech compactification of $S$, which is a compact right topological semigroup, see [7]. Another example is the algebra of almost periodic

(*) Indirizzo dell'A.: Department of Mathematics, Alzahra University, Vanak, Tehran, Iran p.code:1993891176.

School of Mathematics, Institute for Research in Fundamental Sciences (IPM), P.O. Box: 19395-5746, Tehran, Iran.

E-mail: behrouzif@yahoo.com

This research was in part supported by a grant from IPM (No. 90470015). 
functions on a group $G$. Recall that a bounded function $f$ on a group $G$ is said to be almost periodic if the set of all left translations $\left\{L_{x} f: x \in G\right\}$ of $f$ has compact closure in $\ell^{\infty}(G)$, where $L_{x} f$ is defined by $L_{x} f(y)=f(x y)$. This algebra induces the Bohr compactification of $G$ which is denoted by $\mathfrak{b} G$. It is proved that $\mathfrak{b} G$ is a compact group and there exists a homomorphism $\phi: G \rightarrow \mathfrak{b} G$ such that $\phi(G)$ is dense in $\mathfrak{b} G$. In fact $(\mathfrak{b} G, \phi)$ is a group compactification for $G$. This compactification is the biggest compactification, in the sense that if $(K, \psi)$ is another group compactification for $G$, then there exists a continuous homomorphism $T: \mathfrak{b} G \rightarrow K$ such that $T \circ \phi=\psi$. For more details, see [1].

The notion of almost periodic functions on $(\mathbb{R},+)$ was created by Harald Bohr in [3]. The notion is a generalization of purely periodic functions. This notion was extended to arbitrary groups by Bochner and J. Von Neumman in [2] and thereafter this theory was extended to semigroups. For further notes on almost periodic functions see; e.g. [5] and [1].

This note serves two purposes. First, we want to generalize the definition of almost periodic functions on groups to groupoids. The main difficulty in carrying out this construction is that the product operation on a groupoid is partially defined and the definition of translation of a function is not trivially generalized. In Section 3, we define the translation of a function on groupoids and then we define almost periodic functions on groupoids. The second aim of this paper is to obtain a compactification for a groupoid $G$ by showing that the set of all almost periodic functions on $G$, $A P(G)$, is a $\mathrm{C}^{*}$-subalgebra of $\ell^{\infty}(G)$. This fact is shown in Corollary 3.5. Also, we investigate the topological and algebraic properties of the maximal ideal space $A P(G)$. In Section 4, we examine the results in the previous section for groupoids arising from equivalence relations on a set $X$ and in this setting we get a compactification for $X$.

\section{Preliminaries and notations}

Let $X$ be an arbitrary set. We denote by $\ell^{\infty}(X)$ the set of bounded complex-valued functions on $X$. So, $\ell^{\infty}(X)$ with the pointwise operations and the norm

$$
\|f\|_{\infty}=\sup _{x \in X}|f(x)|
$$

is a unital commutative $\mathrm{C}^{*}$-algebra. Let $X$ be a Hausdorff topological space. Then $C_{b}(X)$ stands for the $\mathrm{C}^{*}$-algebra of bounded continuous com- 
plex-valued functions on $X$. We will replace $C_{b}(X)$ by $C(X)$ when $X$ is compact.

In this paper, we are interested in compact subsets of $\ell^{\infty}(X)$. The following lemma which is an easy consequence of Proposition 13.1 in [11], will be frequently applied in the sequel.

Lemma 2.1. Let $\mathcal{C}$ be a subset of $\ell^{\infty}(X)$. Then the closure of $\mathcal{C}$ in $\ell^{\infty}(X)$, $\overline{\mathcal{C}}$, is compact if and only if for any $\varepsilon>0$ there exist subsets $X_{1}, X_{2}, \cdots, X_{n}$ of $X$ such that $X=X_{1} \cup X_{2} \cup \cdots \cup X_{n}$ with the property that if $x, y \in X_{j}$, for some $1 \leq j \leq n$, then $|f(x)-f(y)|<\varepsilon$ for all $f \in \mathcal{C}$.

Here are some elementary definitions in groupoid literature. For more details, we refer the reader to [8], [9] and [10].

A groupoid is a set $G$ endowed with a product map $(x, y) \mapsto$ $x y: G^{(2)} \rightarrow G$ where $G^{(2)}$ is a subset of $G \times G$ called the set of composable pairs, and an inverse map $x \mapsto x^{-1}: G \rightarrow G$ such that the following properties hold:

(i) If $(x, y) \in G^{(2)}$ and $(y, z) \in G^{(2)}$, then $(x y, z) \in G^{(2)},(x, y z) \in G^{(2)}$ and $(x y) z=x(y z)$.

(ii) $\left(x^{-1}\right)^{-1}=x$, for all $x \in G$.

(iii) For all $x \in G,\left(x, x^{-1}\right) \in G^{(2)}$ and if $(z, x) \in G^{(2)}$, then $z\left(x x^{-1}\right)=z$.

(iv) For all $x \in G,\left(x^{-1}, x\right) \in G^{(2)}$ and if $(x, y) \in G^{(2)}$, then $\left(x^{-1} x\right) y=y$.

The maps $r$ and $d$ on $G$, defined by the formulae $r(x)=x x^{-1}$ and $d(x)=x^{-1} x$, are called the range map and the source (domain) map, respectively. A pair $(x, y)$ is composable if and only if $r(y)=d(x)$. The unit space $G^{0}$ is defined by $G^{0}=d(G)=r(G)$. Its elements are units in the sense that $x d(x)=r(x) x=x$. Units will usually be denoted by $u, v, w$ while arbitrary elements will be denoted by $x, y, z$. The fibres of the range and the source maps are denoted by $G^{u}=r^{-1}(\{u\})$ and $G_{v}=d^{-1}(\{v\})$ respectively. Also for $u, v \in G^{0}, G_{u}^{v}=G_{u} \cap G^{v}$.

\section{Generalization of almost periodic functions}

In this section we define almost periodic functions on a groupoid $G$. Since in the usual definition of almost periodic functions on groups the translations of functions play basic role, first we define translations of functions on groupoids. 
Definition 3.1. Suppose that $u \in G^{0}, x \in G_{u}$ and $y \in G^{u}$. For $f \in \ell^{\infty}(G)$ we denote the left $x$-translation and the right $y$-translation of $f$, respectively, by $L_{x}^{u} f$ and $R_{y}^{u} f$ and we define

$$
\begin{array}{ll}
L_{x}^{u} f: G^{u} \rightarrow \mathrm{C}, & R_{y}^{u} f: G_{u} \rightarrow \mathrm{C}, \\
L_{x}^{u} f(t)=f(x t), & R_{y}^{u} f(t)=f(t y) .
\end{array}
$$

Clearly $L_{x}^{u} f \in \ell^{\infty}\left(G^{u}\right), R_{y}^{u} f \in \ell^{\infty}\left(G_{u}\right)$.

THeOREm 3.2. Let $G$ be a groupoid and let $u \in G^{0}$. Then the following properties of $f \in \ell^{\infty}(G)$ are equivalent:

(i) $\overline{\left\{L_{x}^{u} f: x \in G_{u}\right\}}$ is compact in $\ell^{\infty}\left(G^{u}\right)$.

(ii) $\overline{\left\{R_{x}^{u} f: x \in G^{u}\right\}}$ is compact in $\ell^{\infty}\left(G_{u}\right)$.

Proof. (i) $\Rightarrow$ (ii). Suppose that $\overline{\left\{L_{x}^{u} f: x \in G_{u}\right\}}$ is compact in $\ell^{\infty}\left(G^{u}\right)$ and let $\varepsilon>0$. By Lemma 2.1, there exist subsets $X_{1}, X_{2}, \cdots, X_{n}$ of $G^{u}$ such that if $t, t^{\prime} \in X_{j}$ for some $1 \leq j \leq n$, then for any $x \in G_{u}$,

$$
\left|L_{x}^{u} f(t)-L_{x}^{u} f\left(t^{\prime}\right)\right|<\varepsilon .
$$

Now take a fixed $t_{i}$ in the set $X_{i}(i=1,2, \cdots, n)$; hence, for any $t \in G^{u}$, there is some $t_{j} \in G^{u}$ such that $t$ and $t_{j}$ are both in $X_{j}$, accordingly, for any $x \in G_{u}$, we have

$$
\left|f(x t)-f\left(x t_{j}\right)\right|=\left|L_{x}^{u} f(t)-L_{x}^{u} f\left(t_{j}\right)\right|<\varepsilon,
$$

and hence

$$
\left\|R_{t}^{u} f-R_{t_{j}}^{u} f\right\|_{\infty}<\varepsilon .
$$

This argument shows that the set $\left\{R_{x}^{u} f: x \in G^{u}\right\}$ is totally bounded in $\ell^{\infty}\left(G_{u}\right)$. Therefore, $\overline{\left\{R_{x}^{u} f: x \in G^{u}\right\}}$ is compact in $\ell^{\infty}\left(G_{u}\right)$.

(ii) $\Rightarrow$ (i). The proof of this is the same as (i) $\Rightarrow$ (ii).

Definition 3.3. Let $G$ be a groupoid. A function $f$ is said to be almost periodic function if for any $u \in G^{0}, \overline{\left\{L_{g}^{u} f: g \in G_{u}\right\}}$ is compact in $\ell^{\infty}\left(G^{u}\right)$. The set of almost periodic functions on $G$ is denoted by $A P(G)$.

Theorem 3.4. Let $G$ be a groupoid and let $f, g \in A P(G)$. Then

(i) $f+g, f g, \bar{f} \in A P(G)$ and $A P(G)$ contains the constant functions.

(ii) If $\left\{f_{n}\right\}_{n=1}^{\infty}$ is a sequence in $A P(G)$ such that $f_{n} \rightarrow f$ in $\ell^{\infty}(G)$, then $f \in A P(G)$. 
Proof. (i) Let $\varepsilon>0$ and let $u \in G^{0}$. By Lemma 2.1, there exist subsets $X_{1}^{\prime}, X_{2}^{\prime} \cdots, X_{n}^{\prime}$ and $X_{1}^{\prime \prime}, X_{2}^{\prime \prime}, \cdots, X_{m}^{\prime \prime}$ of $G^{u}$ such that $G^{u}=$ $\bigcup_{i=1}^{n} X_{i}^{\prime}=\bigcup_{j=1}^{n} X_{j}^{\prime \prime}$ and if $t, t^{\prime} \in X_{i}$, then for any $x \in G_{u}$,

$$
\left|L_{x}^{u} f(t)-L_{x}^{u} f\left(t^{\prime}\right)\right|<\varepsilon
$$

and if $t, t^{\prime} \in X_{j}^{\prime \prime}$, then for any $x \in G_{u}$,

$$
\left|L_{x}^{u} g(t)-L_{x}^{u} g\left(t^{\prime}\right)\right|<\varepsilon .
$$

Set $X_{i, j}=X_{i}^{\prime} \cap X_{j}^{\prime \prime}(i=1,2, \cdots, n$ and $j=1,2, \cdots, m)$. If $t, t^{\prime} \in X_{i, j}$, then for any $x \in G_{u}$,

$$
\begin{aligned}
\left|L_{x}^{u}(f+g)(t)-L_{x}^{u}(f+g)\left(t^{\prime}\right)\right| & \leq\left|L_{x}^{u} f(t)-L_{x}^{u} f\left(t^{\prime}\right)\right| \\
& +\left|L_{x}^{u} g(t)-L_{x}^{u} g\left(t^{\prime}\right)\right| \\
& <\varepsilon+\varepsilon=2 \varepsilon .
\end{aligned}
$$

Also, we have:

$$
\begin{aligned}
\left|L_{x}^{u}(f g)(t)-L_{x}^{u}(f g)\left(t^{\prime}\right)\right| & =\left|f(x t) g(x t)-f\left(x t^{\prime}\right) g\left(x t^{\prime}\right)\right| \\
& \leq|f(x t)|\left|L_{x}^{u} g(t)-L_{x}^{u} g\left(x t^{\prime}\right)\right| \\
& +\left|g\left(x t^{\prime}\right)\right|\left|L_{x}^{u} f(t)-L_{x}^{u} f\left(x t^{\prime}\right)\right| \\
& \leq\|f\|_{\infty} \varepsilon+\|g\|_{\infty} \varepsilon .
\end{aligned}
$$

Therefore, by Lemma 2.1, the sets $\overline{\left\{L_{x}^{u}(f+g): x \in G_{u}\right\}}$ and $\overline{\left\{L_{x}^{u}(f g): x \in G_{u}\right\}}$ are compact in $\ell^{\infty}\left(G^{u}\right)$.

(ii) Let $\varepsilon>0$. Pick $N \in \mathbb{N}$ such that for any $t \in G,\left|f_{N}(t)-f(t)\right|<\varepsilon$. Since $f_{N} \in A P(G)$, there exist subsets $X_{1}, X_{2}, \cdots, X_{n}$ of $G^{u}$ such that $G^{u}=\bigcup_{i=1}^{n} X_{i}$ and if $t, t^{\prime} \in X_{i}$, then

$$
\left|L_{x}^{u} f_{N}(t)-L_{x}^{u} f_{N}\left(t^{\prime}\right)\right|<\varepsilon .
$$

If $t, t^{\prime} \in X_{i}$, then we have:

$$
\begin{aligned}
\left|L_{x}^{u} f(t)-L_{x}^{u} f\left(t^{\prime}\right)\right| & \leq\left|L_{x}^{u} f(t)-L_{x}^{u} f_{N}(t)\right|+\left|L_{x}^{u} f_{N}(t)-L_{x}^{u} f_{N}\left(t^{\prime}\right)\right| \\
& +\left|L_{x}^{u} f_{N}\left(t^{\prime}\right)-L_{x}^{u} f\left(t^{\prime}\right)\right| \\
& =\left|f(x t)-f_{N}(x t)\right|+\left|L_{x}^{u} f_{N}(t)-L_{x}^{u} f_{N}\left(t^{\prime}\right)\right| \\
& +\left|f_{N}\left(x t^{\prime}\right)-f\left(x t^{\prime}\right)\right| \\
& <\varepsilon+\varepsilon+\varepsilon=3 \varepsilon .
\end{aligned}
$$


Corollary 3.5. Let $G$ be a groupoid. Then $A P(G)$ is a unital $C^{*}$ subalgebra of $\ell^{\infty}(G)$.

Let $\mathfrak{b} G$ be the maximal ideal space of $A P(G)$. Then $\mathfrak{b} G$ is a compact Hausdorff topological space under the Gelfand topology and by the Gelfand-Naimark theorem, $A P(G)$ is isometrically *-isomorphic to $C(\mathfrak{b} G)$. We define $\varphi: G \rightarrow \mathfrak{b} G$ by $\varphi(x)(f)=f(x)$. It is easy to see that $\varphi$ is well-defined and $\varphi(G)$ is dense in $\mathfrak{b} G$.

We can easily extend the functions $L_{x}^{u} f$ and $R_{x}^{u} f$ to whole of $G$. For any $x \in G$, we define

$$
\begin{gathered}
\mathcal{L}_{x} f: G \rightarrow \mathrm{C}, \\
\mathcal{L}_{x} f(t)= \begin{cases}f(x t) & t \in G^{d(x)} \\
0 & t \notin G^{d(x)}\end{cases} \\
\mathcal{R}_{x} f(t)= \begin{cases}f(t x) & t \in G_{r(x)} \\
0 & t \notin G_{r(x)} .\end{cases}
\end{gathered}
$$

Therefore, the functions $\mathcal{L}_{x} f$ and $\mathcal{R}_{x} f$ are in $\ell^{\infty}(G)$ and $\left.\mathcal{L}_{x} f\right|_{G^{d(x)}}=L_{x}^{d(x)} f$ and $\left.\mathcal{R}_{x} f\right|_{G_{r(x)}}=R_{x}^{r(x)} f$.

THeORem 3.6. Let $G$ be a groupoid and let $f \in A P(G)$. Then for any $x \in G$, the functions $\mathcal{L}_{x} f$ and $\mathcal{R}_{x} f$ are in $A P(G)$.

Proof. Let $x_{0} \in G$. A straightforward verification shows that

$$
L_{x}^{u}\left(\mathcal{L}_{x_{0}} f\right)= \begin{cases}L_{x_{0} x}^{u} f & x \in G^{d\left(x_{0}\right)} \\ 0 & x \notin G^{d\left(x_{0}\right)} .\end{cases}
$$

Therefore,

$$
\begin{aligned}
\overline{\left\{L_{x}^{u}\left(\mathcal{L}_{x_{0}} f\right): x \in G_{u}\right\}} & \subseteq \overline{\left\{L_{x_{0} x}^{u} f: x \in G_{u}^{d\left(x_{0}\right)}\right\} \cup\{0\}} \\
& \subseteq \overline{\left\{L_{x}^{u} f: x \in G_{u}\right\}} \cup\{0\} .
\end{aligned}
$$

Since $\overline{\left\{L_{x}^{u} f: x \in G_{u}\right\}} \cup\{0\}$ is compact so is $\overline{\left\{L_{x}^{u}\left(\mathcal{L}_{x_{0}} f\right): x \in G_{u}\right\}}$. Similarly, it is proved that $\mathcal{R}_{x} f$ is in $A P(G)$.

Let $\eta \in \mathfrak{b} G$ and let $f \in A P(G)$. We define two functions $T_{\eta, f}: G \rightarrow \mathrm{C}$ and $S_{\eta, f}: G \rightarrow$ C by

$$
T_{\eta, f}(x)=\eta\left(\mathcal{L}_{x} f\right) \quad, \quad S_{\eta, f}(x)=\eta\left(\mathcal{R}_{x} f\right) .
$$

By Theorem 3.6, the functions $T_{\eta, f}$ and $S_{\eta, f}$ are well-defined and are in $\ell^{\infty}(G)$. 
THEOREM 3.7. Let $G$ be a groupoid and $f \in A P(G)$.

(i) If $\eta \in \overline{\varphi\left(G^{u}\right)}$ for some $u \in G^{0}$, then $T_{\eta, f} \in A P(G)$.

(ii) If $\eta \in \overline{\varphi\left(G_{u}\right)}$ for some $u \in G^{0}$, then $S_{\eta, f} \in A P(G)$.

Proof. (i) Let $\left\{x_{\alpha}\right\}_{\alpha \in I}$ be a net in $G^{u}$ such that $\varphi\left(x_{\alpha}\right) \rightarrow \eta$. Hence, $\left.T_{\varphi\left(x_{\alpha}\right), f}\right|_{G_{u}}=R_{x_{\alpha}}^{u} f=\left.\mathcal{R}_{x_{\alpha}} f\right|_{G_{u}}$ and $\left.\mathcal{R}_{x_{\alpha}} f\right|_{G_{u}}$ is pointwise convergent to $\left.T_{\eta, f}\right|_{G_{u}}$. Since the closure of $\left\{R_{x}^{u} f: x \in G^{u}\right\}$ under the norm topology is compact, the closure of $\left\{R_{x}^{u} f: x \in G^{u}\right\}$ under the norm topology is the same as the closure of $\left\{R_{x}^{u} f: x \in G^{u}\right\}$ under the pointwise topology. Therefore, $\left.T_{\eta, f}\right|_{G_{u}} \in \overline{\left\{R_{x}^{u} f: x \in G^{u}\right\}}$ and hence $\left.\mathcal{R}_{x_{\alpha}} f\right|_{G_{u}}$ is norm convergent to $\left.T_{\eta, f}\right|_{G_{u}}$ in $\ell^{\infty}\left(G_{u}\right)$. On the other hand, if $t \notin G_{u}$, then $T_{\eta, f}(t)=\mathcal{R}_{x_{\alpha}} f(t)=0$, thus $\mathcal{R}_{x_{\alpha}} f$ is norm convergent to $T_{\eta, f}$. By Theorem 3.6 and Corollary 3.5, $T_{\eta, f} \in A P(G)$.

(ii) The proof is similar to (i).

Corollary 3.8. Let $G$ be a groupoid. If $u, v \in G^{0}$ and $u \neq v$, then $\overline{\varphi\left(G^{u}\right)} \cap \overline{\varphi\left(G^{v}\right)}=\emptyset$.

Proof. Let $\eta \in \overline{\varphi\left(G^{u}\right)} \cap \overline{\varphi\left(G^{v}\right)}$. Choose $f \in A P(G)$ with $\eta(f)=1$. Since $v \notin G^{u}, T_{\eta, f}(v)=0$. Let $\left\{x_{\alpha}\right\}_{I}$ be a net in $G^{v}$ such that $\varphi\left(x_{\alpha}\right) \rightarrow \eta$. So

$$
\begin{aligned}
T_{\eta, f}(v) & =\lim _{\alpha} T_{\varphi\left(x_{\alpha}\right), f}(v) \\
& =\lim _{\alpha} f\left(x_{\alpha}\right) \\
& =\lim _{\alpha} \varphi\left(x_{\alpha}\right)(f)=\eta(f)=1 .
\end{aligned}
$$

This is a contradiction.

Corollary 3.9. Let $G$ be a groupoid and let $u \in G^{0}$. Suppose that $f \in A P(G)$.

(i) If $\eta \in \overline{\varphi\left(G^{u}\right)}$ and $\eta_{\alpha} \in \overline{\varphi\left(G^{u}\right)}$ with $\eta_{\alpha} \rightarrow \eta$, then $T_{\eta_{\alpha, f}} \rightarrow T_{\eta, f}$ in $A P(G)$.

(ii) If $\eta \in \overline{\varphi\left(G_{u}\right)}$ and $\eta_{\alpha} \in \overline{\varphi\left(G_{u}\right)}$ with $\eta_{\alpha} \rightarrow \eta$, then $S_{\eta_{\alpha, f}} \rightarrow S_{\eta, f}$ in $A P(G)$.

Proof. (i) By Theorem 3.7, $T_{\eta_{\alpha, f}}$ and $T_{\eta, f}$ are in $A P(G)$, hence $\left.T_{\eta_{\alpha, f}}\right|_{G_{u}},\left.T_{\eta, f}\right|_{G_{u}}$ are in $\overline{\left\{R_{x}^{u}: x \in G^{u}\right\}}$. Since $T_{\eta_{\alpha, f}}$ is pointwise convergent to $T_{\eta, f}$ in $\ell^{\infty}(G)$ and $\overline{\left\{R_{x}^{u}: x \in G^{u}\right\}}$ is compact, thus $\left.T_{\eta_{x, f}}\right|_{G_{u}}$ converges to $\left.T_{\eta, f}\right|_{G_{u}}$ in the norm topology of $\ell^{\infty}\left(G_{u}\right)$. On the other hand, $\left.T_{\eta_{\alpha, f}}\right|_{\left(G \backslash G_{u}\right)}=$ 
$\left.T_{\eta, f}\right|_{\left(G \backslash G_{u}\right)}=0$. Therefore, $T_{\eta_{\alpha, f}}$ converges to $T_{\eta, f}$ in the norm topology of $A P(G)$.

(ii) The proof is similar to (i).

THEOREM 3.10. Let $G$ be a groupoid and $\eta, \theta \in \mathfrak{b} G$. Let $\left\{x_{\alpha}\right\}_{I}$ and $\left\{y_{\beta}\right\}_{J}$ be nets in $G_{u}$ and $G^{u}$, respectively, such that $\varphi\left(x_{\alpha}\right) \rightarrow \eta$ and $\varphi\left(y_{\beta}\right) \rightarrow \theta$. Then

$$
\lim _{\alpha} \lim _{\beta} \varphi\left(x_{\alpha} y_{\beta}\right)=\lim _{\beta} \lim _{\alpha} \varphi\left(x_{\alpha} y_{\beta}\right) .
$$

Proof. Let $f \in A P(G)$. We have:

$$
\begin{aligned}
\lim _{\alpha} \lim _{\beta} \varphi\left(x_{\alpha} y_{\beta}\right)(f) & =\lim _{\alpha} \lim _{\beta} f\left(x_{\alpha} y_{\beta}\right) \\
& =\lim _{\alpha} \lim _{\beta} \varphi\left(y_{\beta}\right)\left(\mathcal{L}_{x_{\alpha}} f\right) \\
& =\lim _{\alpha} \theta\left(\mathcal{L}_{x_{\alpha}} f\right)=\lim _{\alpha} \varphi\left(x_{\alpha}\right)\left(T_{\theta, f}\right) \\
& =\eta\left(T_{\theta, f}\right) .
\end{aligned}
$$

By Corollary 3.9 (i), $T_{\varphi\left(y_{\beta}\right), f} \rightarrow T_{\theta, f}$ in $A P(G)$. Therefore,

$$
\begin{aligned}
\eta\left(T_{\theta, f}\right) & =\lim _{\beta} \eta\left(T_{\varphi\left(y_{\beta}\right), f}\right) \\
& =\lim _{\beta} \lim _{\alpha} \varphi\left(x_{\alpha}\right)\left(T_{\varphi\left(y_{\beta}\right), f}\right) \\
& =\lim _{\beta} \lim _{\alpha} f\left(x_{\alpha} y_{\beta}\right) \\
& =\lim _{\beta} \lim _{\alpha} \varphi\left(x_{\alpha} y_{\beta}\right)(f) .
\end{aligned}
$$

Corollary 3.11. Let $G$ be a groupoid. If $\eta \in \overline{\varphi\left(G_{u}\right)}$ and $\theta \in \overline{\varphi\left(G^{u}\right)}$, then

$$
\eta\left(T_{\theta, f}\right)=\theta\left(S_{\eta, f}\right)
$$

Proof. We have:

$$
\begin{aligned}
\eta\left(T_{\theta, f}\right) & =\lim _{\beta} \lim _{\alpha} \varphi\left(x_{\alpha} y_{\beta}\right)(f) \\
& =\lim _{\beta} \lim _{\alpha} \varphi\left(x_{\alpha}\right)\left(\mathcal{R}_{y_{\beta}} f\right) \\
& =\lim _{\beta} \eta\left(\mathcal{R}_{y_{\beta}} f\right)=\lim _{\beta} \varphi\left(y_{\beta}\right)\left(S_{\eta, f}\right) \\
& =\theta\left(S_{\eta, f}\right) .
\end{aligned}
$$


For a groupoid $G$, set

$$
(\mathfrak{b} G)_{0}^{(2)}=\bigcup_{u \in G^{0}} \overline{\varphi\left(G_{u}\right)} \times \overline{\varphi\left(G^{u}\right)}, \quad(\mathfrak{b} G)_{(0)}=\bigcup_{u, v \in G^{0}} \overline{\varphi\left(G_{u}^{v}\right)},
$$

and

$$
(\mathfrak{b} G)_{1}^{(2)}=\bigcup_{u, v, w \in G^{0}} \overline{\varphi\left(G_{u}^{v}\right)} \times \overline{\varphi\left(G_{w}^{u}\right)} .
$$

We define an operation “*” on $(\mathfrak{b} G)_{0}^{(2)}$ by $\eta * \theta(f)=\eta\left(T_{\theta, f}\right)$, where $(\eta, \theta) \in$ $(\mathfrak{b} G)_{0}^{(2)}$.

Theorem 3.12. Let $G$ be a groupoid and $u \in G^{0}$. Then the map $(\eta, \theta) \mapsto \eta * \theta: \overline{\varphi\left(G_{u}\right)} \times \overline{\varphi\left(G^{u}\right)} \rightarrow \mathfrak{b} G$ is continuous.

Proof. Let $\varepsilon>0, f \in A P(G)$ and $\left(\eta_{0}, \theta_{0}\right) \in \overline{\varphi\left(G_{u}\right)} \times \overline{\varphi\left(G^{u}\right)}$. Let $V=$ $\left\{\gamma \in \mathfrak{b} G:\left|\gamma(f)-\eta_{0} * \theta_{0}(f)\right|<\varepsilon\right\}$. By Corollary 3.9 (i), the map $\theta \mapsto T_{\theta, f}: \varphi\left(G^{u}\right) \rightarrow A P(G)$ is norm continuous. Therefore, there exists an open set $U_{1}$ in $\mathfrak{b} G$ containing $\theta_{0}$ such that for any $\theta \in U_{1}$, $\left\|T_{\theta, f}-T_{\theta_{0}, f}\right\|<\varepsilon / 2$. Let $U_{2}=\left\{\gamma \in \mathfrak{b} G:\left|\gamma\left(T_{\theta_{0}, f}\right)-\eta_{0}\left(T_{\theta_{0}, f}\right)\right|<\varepsilon / 2\right\}$. For $(\eta, \theta) \in U_{2} \times U_{1}$,

$$
\begin{aligned}
\left|\eta * \theta(f)-\eta_{0} * \theta_{0}(f)\right| & =\left|\eta\left(T_{\theta, f}\right)-\eta_{0}\left(T_{\theta_{0}, f}\right)\right| \\
& \leq\|\eta\| .\left\|T_{\theta, f}-T_{\theta_{0}, f}\right\|+\left|\eta\left(T_{\theta_{0}, f}\right)-\eta_{0}\left(T_{\theta_{0}, f}\right)\right| \\
& \leq \varepsilon .
\end{aligned}
$$

Let $K$ be a set and let $K^{(2)}$ be a subset of $K \times K$. If $f \in K$, we set

$$
K_{f}=\left\{g \in K:(g, f) \in K^{(2)}\right\}, \quad K^{f}=\left\{g \in K:(f, g) \in K^{(2)}\right\} .
$$

Definition 3.13. A Jordan semigroupoid is a triple $\left(K, K^{(2)}, *\right)$, such that $K$ is a set, $K^{(2)}$ is a subset of $K \times K$ and $*$ is an operation from $K^{(2)}$ to $K$.

Let $\left(\Lambda, \Lambda^{(2)}, *\right)$ and $\left(\Lambda^{\prime}, \Lambda^{\prime(2)}, *^{\prime}\right)$ be Jordan semigroupoids. A map $T: \Lambda \rightarrow \Lambda^{\prime}$ is called homomorphism if $(f, g) \in \Lambda^{(2)}$, then $(T(f), T(g)) \in \Lambda^{\prime(2)}$ and $T(f * g)=T(f) *^{\prime} T(g)$.

THeorem 3.14. Let $G$ be a groupoid and let $\left(K, K^{(2)}, \star\right)$ be a Jordan semigroupoid such that $K$ is compact Hausdorff topological space. Suppose that

(i) For any $f \in K$, the map $g \mapsto g f: K_{f} \rightarrow K$ and the map $g \mapsto f g: K^{f} \rightarrow K$ are continuous, 
(ii) $\psi: G \rightarrow K$ is a homomorphism such that for any $u \in G^{0}$, $\overline{\psi\left(G_{u}\right)} \times \overline{\psi\left(G^{u}\right)} \subseteq K^{(2)}$.

Then there exists a unique continuous homomorphism $\Phi: \mathfrak{b} G \rightarrow K$ such that $\Phi \circ \varphi=\psi$.

Proof. Let $F \in C(K)$. We want to show that $F \circ \psi \in A P(G)$. To do this, consider the map $(t, s) \mapsto F(\psi(s t)): \overline{\psi\left(G_{u}\right)} \times \overline{\psi\left(G^{u}\right)} \rightarrow$ C. By assumption, this map is separately continuous and hence the map $x \mapsto L_{s} F: \overline{\psi\left(G_{u}\right)} \rightarrow$ $C\left(\overline{\psi\left(G^{u}\right)}\right)$ is norm continuous; see [1, Lemma B.3]. By continuity, the set $\left\{L_{s} F: s \in \overline{\psi\left(G_{u}\right)}\right\}$ is a compact subset of $C\left(\overline{\psi\left(G^{u}\right)}\right)$ and hence the set $\left\{L_{\psi(x)} F: x \in G_{u}\right\}$ has compact closure in $C\left(\overline{\psi\left(G^{u}\right)}\right)$. So, by applying Lemma 2.1, we conclude that the set $\left\{L_{x}(F \circ \psi): x \in G_{u}\right\}$ has compact closure in $\ell^{\infty}\left(G^{u}\right)$, as required. Then it is easy to verify that the map $T: C(K) \rightarrow$ $A P(G)$ defined by $T(F)=F \circ \psi$ is a *-homomorphism. If $\Phi=\left.T^{*}\right|_{\mathfrak{b} G}$, then $\Phi: \mathfrak{b} G \rightarrow K$ is continuous and $\Phi \circ \varphi=\psi$. For the last equality, suppose that $x \in G$. For any $F \in C(K)$, we have:

$$
F(\Phi(\varphi(x)))=\varphi(x)(T(F))=F \circ \psi(x)=F(\psi(x)) .
$$

Therefore, $\Phi \circ \varphi=\psi$. Now, assume that $(\eta, \theta) \in \overline{\varphi\left(G_{u}\right)} \times \overline{\varphi\left(G^{u}\right)}$ for some $u \in G^{0}$. Let $\left\{x_{\alpha}\right\}_{I}$ and $\left\{y_{\beta}\right\}_{J}$ be nets in $G_{u}$ and $G^{u}$, respectively, such that $\varphi\left(x_{\alpha}\right) \rightarrow \eta$ and $\varphi\left(y_{\beta}\right) \rightarrow \theta$. Then

$$
\Phi(\eta)=\lim _{\alpha} \Phi\left(\varphi\left(x_{\alpha}\right)\right)=\lim _{\alpha} \psi\left(x_{\alpha}\right) .
$$

So $\Phi(\eta) \in \overline{\psi\left(G_{u}\right)}$. Similarly, $\Phi(\theta) \in \overline{\psi\left(G^{u}\right)}$, and hence $(\Phi(\eta), \Phi(\theta)) \in \overline{\psi\left(G_{u}\right)} \times$ $\overline{\psi\left(G^{u}\right)} \subseteq K^{(2)}$. Also, we have:

$$
\begin{aligned}
\Phi(\eta) \star \Phi(\theta) & =\lim _{\alpha} \lim _{\beta} \Phi\left(\varphi\left(x_{\alpha}\right)\right) \star \Phi\left(\varphi\left(y_{\beta}\right)\right) \\
& =\lim _{\alpha} \lim _{\beta} \psi\left(x_{\alpha}\right) \star \psi\left(y_{\beta}\right) \\
& =\lim _{\alpha} \lim _{\beta} \psi\left(x_{\alpha} y_{\beta}\right) \\
& =\lim _{\alpha} \lim _{\beta} \Phi\left(\varphi\left(x_{\alpha} y_{\beta}\right)\right)=\Phi(\eta * \theta) .
\end{aligned}
$$

THEOREM 3.15. Suppose that $G$ is a groupoid and $(\eta, \theta) \in(\mathfrak{b} G)_{0}^{(2)}$.

(i) Let $\theta \in(\mathfrak{b} G)_{(0)}$. Then $\eta * \theta \in \overline{\varphi\left(G_{v}\right)}$ if and only if $\theta \in \overline{\varphi\left(G_{v}^{u}\right)}$ for some $u \in G^{0}$.

(ii) Let $\eta \in(\mathfrak{b} G)_{(0)}$. Then $\eta * \theta \in \overline{\varphi\left(G^{w}\right)}$ if and only if $\eta \in \overline{\varphi\left(G_{u}^{w}\right)}$ for some $u \in G^{0}$. 
Proof. (i) Suppose that $\theta \in \overline{\varphi\left(G_{v}^{u}\right)}$ and $\left\{x_{\alpha}\right\}_{\alpha \in J}$ and $\left\{y_{\alpha}\right\}_{\alpha \in J}$ are nets in $G_{u}$ and $G_{v}^{u}$, respectively, such that $\varphi\left(x_{\alpha}\right) \rightarrow \eta$ and $\varphi\left(y_{\alpha}\right) \rightarrow \theta$. Then $x_{\alpha} y_{\alpha} \in G_{v}$ and hence $\eta * \theta=\lim _{\alpha} \varphi\left(x_{\alpha}\right) \varphi\left(y_{\alpha}\right) \in \overline{\varphi\left(G_{v}\right)}$. Conversely, let $\eta * \theta \in \overline{\varphi\left(G_{v}\right)}$ and $\theta \in \overline{\varphi\left(G_{v^{\prime}}^{u}\right)}$. This implies that $\eta * \theta \in \overline{\varphi\left(G_{v}\right)} \cap \overline{\varphi\left(G_{v^{\prime}}\right)}$. By Corollary 3.5, $v=v^{\prime}$.

(ii) The proof is similar to (i).

Let $G$ be a groupoid. If $f \in A P(G)$, define $\widehat{f}: G \longrightarrow$ C by $\widehat{f}(x)=f\left(x^{-1}\right)$. It is easy to see that $\widehat{f} \in A P(G)$. We extend the inverse operation " -1 " on $G$ to an involution on $\mathfrak{b} G$. If $\eta \in \mathfrak{b} G$, define $\eta^{-1}(f)=\eta(\widehat{f})$. Since $\widehat{f g}=\widehat{f} \widehat{g}$, $\eta^{-1} \in \mathfrak{b} G$ and the map $\eta \mapsto \eta^{-1}$ is continuous. Also, if $\eta \in \overline{\phi\left(G_{v}^{u}\right)}$, then by Theorem 3.12, $\eta^{-1} * \eta=v$ and $\eta * \eta^{-1}=u$.

Definition 3.16. Let $\Lambda$ be a set and $\Lambda^{(2)}$ be a subset of $\Lambda \times \Lambda$. An operation $*: \Lambda^{(2)} \longrightarrow \Lambda$ is called to be associative if $f, g, h \in \Lambda$ are such that either

(i) $(f, g) \in \Lambda^{(2)}$ and $(g, h) \in \Lambda^{(2)}$, or

(ii) $(f, g) \in \Lambda^{(2)}$ and $(f * g, h) \in \Lambda^{(2)}$, or

(iii) $(g, h) \in \Lambda^{(2)}$ and $(f, g * h) \in \Lambda^{(2)}$

then all $(f, g),(g, h),(f * g, h)$ and $(f, g * h)$ lie in $\Lambda^{(2)}$, and

$$
(f * g) * h=f *(g * h) .
$$

Theorem 3.17. Let G be a groupoid. Then

(i) the operation $*:(\mathfrak{b} G)_{1}^{(2)} \longrightarrow \mathfrak{b} G$ is associative.

(ii) if $\eta \in \overline{\varphi\left(G_{u}\right)}$, then $\eta * u=\eta$.

(iii) if $\eta \in(\mathfrak{b} G)_{(0)}$, then $\left(\eta, \eta^{-1}\right) \in(\mathfrak{b} G)_{1}^{(2)}$ and $\left(\eta, \eta^{-1}\right) \in G^{(0)}$.

\section{Equivalence relations as groupoids}

Let $X$ be any set and let $\mathcal{R}$ be an equivalence relation on $X$. Set

$$
\mathcal{R}^{(2)}=\{((x, y),(y, z)):(x, y),(y, z) \in \mathcal{R}\},
$$

define $(x, y)(y, z)=(x, z)$ and define $(x, y)^{-1}=(y, x)$. Then it is easy to check that $\mathcal{R}$ becomes a groupoid and it is evident that $\mathcal{R}^{(0)}$ is the diagonal $\Delta=\{(x, x): x \in X\}$. Also, for $x \in X$,

$$
G^{(x, x)}=\{x\} \times[x], \quad G_{(x, x)}=[x] \times\{x\}
$$


where $[x]$ is used to designate the equivalence class determined by $x$. Two extreme cases are to be singled out. If $\mathcal{R}=X \times X$, then $\mathcal{R}$ is called the trivial groupoid on $X$, while if $\mathcal{R}=\Delta$, then $\mathcal{R}$ is called the co-trivial groupoid on $X$.

Let $\mathcal{R}$ be an equivalence relation on a set $X$. So, $f \in \ell^{\infty}(\mathcal{R})$ is almost periodic if and only if for any $x \in X$, the set $\overline{\left\{f_{s}: s \in[x]\right\}}$ is compact in $\ell^{\infty}([x])$, where $f_{s}:[x] \rightarrow \mathbb{C}$ is defined by $f_{s}(t)=f(s, t)$.

Definition 4.1. Let $X$ be any set. A function $f \in \ell^{\infty}(X \times X)$ is called piecewise cylindrical if there exists a partition $X_{1}, X_{2}, \cdots, X_{n}$ for $X$ such that for any $1 \leq k \leq n, s \in X$ and $t, t^{\prime} \in X_{k} \operatorname{imply} f(s, t)=f\left(s, t^{\prime}\right)$. We denote the set of piecewise cylindrical functions on $X \times X$ by $P C(X)$.

THEOREM 4.2. Let $X$ be any set and let $\mathcal{R}$ be an equivalence relation on the set $X$. Then for any $x \in X, P C([x])$ is dense in $\left.A P(\mathcal{R})\right|_{[x] \times[x]}$.

Proof. First, we show that $P C([x])$ is contained in $\left.A P(\mathcal{R})\right|_{[x] \times[x]}$. Suppose that $F \in P C([x])$. Thus there exists a partition $X_{1}, X_{2}, \cdots, X_{n}$ for $[x]$ such that for any $s \in[x]$ and for any $1 \leq k \leq n$ the identity $F(s, t)=F\left(s, t^{\prime}\right)$ holds for all $t, t^{\prime} \in X_{k}$. If we define $f^{0}: \mathcal{R} \rightarrow \mathrm{C}$ by

$$
f^{0}(s, t)= \begin{cases}F(s, t) & (s, t) \in[x] \times[x] \\ 0 & (s, t) \notin[x] \times[x],\end{cases}
$$

then $\left.f^{0}\right|_{[x] \times[x]}=F$. We claim that $f^{0} \in A P(\mathcal{R})$. Let $\varepsilon>0$ and $y \in X$. Consider subsets $X_{1}, X_{2}, \cdots, X_{n}, X_{n+1}=X \backslash[x]$. By assumption, if $t, t^{\prime} \in X_{k}$, then for all $s \in[y],\left|f^{0}(s, t)-f^{0}\left(s, t^{\prime}\right)\right|=0<\varepsilon$. Therefore, by Lemma 2.1 the set $\overline{\left\{f_{s}^{0}: s \in[y]\right\}}$ is compact in $\ell^{\infty}([y])$. Thus $f^{0}$ is almost periodic. Now, suppose that $f \in A P(\mathcal{R})$ and suppose that $\varepsilon>0$. By Lemma 2.1, there exists a partition $X_{1}, X_{2}, \cdots, X_{n}$ for $[x]$ such that if $t, t^{\prime} \in X_{k}$ for some $1 \leq k \leq n$, then for all $s \in[x],\left|f(s, t)-f\left(s, t^{\prime}\right)\right|<\varepsilon$. Let $t_{1} \in X_{1}, t_{2} \in X_{2}, \cdots, t_{n} \in X_{n}$ be fixed points. Define $F:[x] \times[x] \rightarrow \mathbb{C}$ by $F_{0}(s, t)=f\left(s, t_{k}\right)$ if $t \in X_{k}$. Trivially, $F_{0} \in P C([x])$ and if $t \in X_{k}$ for some $1 \leq k \leq n$, then

$$
\left|F_{0}(s, t)-f(s, t)\right|=\left|f\left(s, t_{k}\right)-f(s, t)\right|<\varepsilon .
$$

Let $\mathcal{R}$ be the co-trivial groupoid $\Delta$. Trivially, every $f \in \ell^{\infty}(\mathcal{R})$ is almost periodic. So, $A P(\mathcal{R})=\ell^{\infty}(\mathcal{R})$ and hence $\mathfrak{b} \mathcal{R}=\beta \Delta$. More generally, if $\mathcal{R}$ is an equivalence relation on a set $X$ such that every equivalence class of $\mathcal{R}$ is finite, then $A P(\mathcal{R})=\ell^{\infty}(\mathcal{R})$ and hence $\mathfrak{b} \mathcal{R}=\beta \Delta$. 
THEOREM 4.3. Let $\mathcal{R}$ be the trivial groupoid on a set $X$. Then $\mathfrak{b} \mathcal{R}=$ $\beta X \times \beta X$.

Proof. We prove the theorem in the following steps.

Step 1. If $\left\{x_{\alpha}\right\}_{\alpha \in J},\left\{y_{\alpha}\right\}_{\alpha \in J},\left\{x_{\alpha}^{\prime}\right\}_{\alpha \in J}$ and $\left\{y_{\alpha}^{\prime}\right\}_{\alpha \in J}$ are nets in $X$ such that $\lim _{\alpha} x_{\alpha}=\lim _{\alpha} x_{\alpha}^{\prime}$ and $\lim _{\alpha} y_{\alpha}=\lim _{\alpha} y_{\alpha}^{\prime}$ (the limits are taken in the $\beta X$ ), then $\lim _{\alpha} \varphi\left(x_{\alpha}, y_{\alpha}\right)=\lim _{\alpha} \varphi\left(x_{\alpha}^{\prime}, y_{\alpha}^{\prime}\right)$.

To show that $\lim _{\alpha} \varphi\left(x_{\alpha}, y_{\alpha}\right)=\lim _{\alpha} \varphi\left(x_{\alpha}^{\prime}, y_{\alpha}^{\prime}\right)$ in $\mathfrak{b} \mathcal{R}$, it is enough to show that for every $f \in P C(X)$,

$$
\lim _{\alpha} \varphi\left(x_{\alpha}, y_{\alpha}\right)(f)=\lim _{\alpha} \varphi\left(x_{\alpha}^{\prime}, y_{\alpha}^{\prime}\right)(f) .
$$

Let $X_{1}, X_{2}, \cdots, X_{n}$ be a partition for $X$ such that if $t, t^{\prime} \in X_{k}$ for some $1 \leq k \leq n$, then for any $s \in X, f(s, t)=f\left(s, t^{\prime}\right)$. By Theorem 6.5 in [6], the sets $\overline{X_{1}}, \overline{X_{2}}, \cdots, \overline{X_{n}}$ (the closures are taken in $\beta X$ ) are open and disjoint sets, so, we can assume that there exists $1 \leq j \leq n$ such that for any $\alpha \in J$, $y_{\alpha}, y_{\alpha}^{\prime} \in X_{j}$. Suppose that $x_{\alpha} \rightarrow \theta$ in $\beta X$ and $t^{*}$ is a fixed element in $X_{j}$. Define $g(s)=f\left(s, t^{*}\right)$. Thus $g \in \ell^{\infty}(X)$ and we have:

$$
\begin{aligned}
\lim _{\alpha} \varphi\left(x_{\alpha}, y_{\alpha}\right)(f) & =\lim _{\alpha} f\left(x_{\alpha}, y_{\alpha}\right) \\
& =\lim _{\alpha} f\left(x_{\alpha}, t^{*}\right)=\lim _{\alpha} g\left(x_{\alpha}\right) \\
& =\theta(g) .
\end{aligned}
$$

Similarly, $\lim _{\alpha} \varphi\left(x_{\alpha}^{\prime}, y_{\alpha}^{\prime}\right)(f)=\theta(g)$.

Step 2. If $\left\{\varphi\left(x_{\alpha}, y_{\alpha}\right)\right\}_{\alpha \in J}$ and $\left\{\varphi\left(x_{\alpha}^{\prime}, y_{\alpha}^{\prime}\right)\right\}_{\alpha \in J}$ are nets in $\mathfrak{b} \mathcal{R}$ such that $\lim _{\alpha} \varphi\left(x_{\alpha}, y_{\alpha}\right)=\lim _{\alpha} \varphi\left(x_{\alpha}^{\prime}, y_{\alpha}^{\prime}\right)$, then $\lim _{\alpha} x_{\alpha}=\lim _{\alpha} x_{\alpha}^{\prime}$ and $\lim _{\alpha} y_{\alpha}=\lim _{\alpha} y_{\alpha}^{\prime}$.

Let $g \in \ell^{\infty}(X)$. By Lemma 4.2 the map $f$ defined by $f(s, t)=g(s)$ is an almost periodic function on $\mathcal{R}$. So

$$
\begin{aligned}
\lim _{\alpha} g\left(x_{\alpha}\right) & =\lim _{\alpha} \varphi\left(x_{\alpha}, y_{\alpha}\right)(f) \\
& =\lim _{\alpha} \varphi\left(x_{\alpha}^{\prime}, y_{\alpha}^{\prime}\right)(f) \\
& =\lim _{\alpha} g\left(x_{\alpha}^{\prime}\right) .
\end{aligned}
$$

Thus $\lim _{\alpha} x_{\alpha}=\lim _{\alpha} x_{\alpha}^{\prime}$. By the same proof, $\lim _{\alpha} y_{\alpha}=\lim _{\alpha} y_{\alpha}^{\prime}$.

Let $i_{\beta}: X \rightarrow \beta X$ be the injection map. Therefore, $\beta X \times \beta X$ is compact semigroupoid (in fact it is groupoid as trivial groupoid on $\beta X$ ) and 
$i_{\beta} \times i_{\beta}: X \times X \rightarrow \beta X \times \beta X$ is a continuous homomorphism which satisfies all the conditions of Theorem 3.14. So, there exists a homomorphism $\psi: \mathfrak{b}(X \times X) \rightarrow \beta X \times \beta X$ such that $\psi \circ \varphi=i_{\beta} \times i_{\beta}$. We show that $\psi$ is an isomorphism. Let $\theta_{1}$ and $\theta_{2}$ be in $\mathfrak{b}(X \times X)$ such that $\psi\left(\theta_{1}\right)=\psi\left(\theta_{2}\right)$. Let $\left\{x_{\alpha}\right\}_{\alpha \in J},\left\{y_{\alpha}\right\}_{\alpha \in J},\left\{x_{\alpha}^{\prime}\right\}_{\alpha \in J}$ and $\left\{y_{\alpha}^{\prime}\right\}_{\alpha \in J}$ be nets in $X$ such that $\lim _{\alpha} \varphi\left(x_{\alpha}, y_{\alpha}\right)=\theta_{1}$ and $\lim _{\alpha} \varphi\left(x_{\alpha}^{\prime}, y_{\alpha}^{\prime}\right)=\theta_{2}$. We have:

$$
\begin{aligned}
\lim _{\alpha} i_{\beta} \times i_{\beta}\left(x_{\alpha}, y_{\alpha}\right) & =\lim _{\alpha} \psi \circ \varphi\left(x_{\alpha}, y_{\alpha}\right) \\
& =\psi\left(\theta_{1}\right)=\psi\left(\theta_{2}\right) \\
& =\lim _{\alpha} \psi \circ \varphi\left(x_{\alpha}^{\prime}, y_{\alpha}^{\prime}\right) \\
& =\lim _{\alpha} i_{\beta} \times i_{\beta}\left(x_{\alpha}^{\prime}, y_{\alpha}^{\prime}\right) .
\end{aligned}
$$

Therefore, $\lim _{\alpha} x_{\alpha}=\lim _{\alpha} x_{\alpha}^{\prime}$ and $\lim _{\alpha} y_{\alpha}=\lim _{\alpha} y_{\alpha}^{\prime}$. Thus

$$
\theta_{1}=\lim _{\alpha} \varphi\left(x_{\alpha}, y_{\alpha}\right)=\lim _{\alpha} \varphi\left(x_{\alpha}^{\prime}, y_{\alpha}^{\prime}\right)=\theta_{2} .
$$

Now, suppose that $\left(\gamma_{1}, \gamma_{2}\right) \in \beta X \times \beta X$ and suppose that $\left\{x_{\alpha}\right\}$ and $\left\{y_{\alpha}\right\}$ are nets in $\beta X$ such that $x_{\alpha} \rightarrow \gamma_{1}$ and $y_{\alpha} \rightarrow \gamma_{2}$. By passing to a subsequence, we may assume that $\varphi\left(x_{\alpha}, y_{\alpha}\right)$ is convergent in $\mathfrak{b}(X \times X)$. Let $\varphi\left(x_{\alpha}, y_{\alpha}\right) \rightarrow \theta$. We have:

$$
\psi(\theta)=\lim _{\alpha} \psi \circ \varphi\left(x_{\alpha}, y_{\alpha}\right)=\lim _{\alpha} i_{\beta} \times i_{\beta}\left(x_{\alpha}, y_{\alpha}\right)=\left(\gamma_{1}, \gamma_{2}\right) .
$$

REMARK 4.4. Let $\mathcal{R}$ be an equivalence relation on a set $X$ and let $\mathfrak{b} \delta: X \rightarrow \mathfrak{b} \mathcal{R}$ be a map defined by $\mathfrak{b} \delta(x)=\varphi(x, x)$. Suppose that $\mathfrak{b} X$ is the closure of the image $X$ under $\mathfrak{b} \delta$. Then $(\mathfrak{b} X, \mathfrak{b} \delta)$ is a compactification for $X$ which is called $\mathcal{R}$-almost periodic compactification.

Acknowledgments. The authors are grateful to the referee for careful reading of the paper, and for providing several helpful suggestions for improvement in the article.

\section{REFERENCES}

[1] J. F. Berglund - H.D. Junghenn - P. Milnes: Analysis on Semigroups: Function Spaces, Compactifications, Representations. Wiley, New York, 1989.

[2] Bochner - J. Von Neumann, Almost periodic functions in groups, Trans. Amer. Math. Soc. 37 (1935), 21-50.

[3] H. BoHr, Zur Theorie der fastperiodischen Funcktionen, I, II, III, Acta Math. 45 (1924), 29-127, 46 (1925), 101-214, 47 (1926), 237-281, 
[4] A. BRowder, Introduction to function algebras, W. A. Benjamin, New York, 1969.

[5] J. Dixmier, C*-Algebras, North-Holland, Amsterdam, 1977.

[6] L. Gillman - M. Jerison, Rings of continuous functions, van Nostrand, Princeton, 1960.

[7] N. Hindman - D. Strauss, Algebra in the Stone-Čech Compactification: Theory and Applications, Walter de Gruyter, Berlin, 1998.

[8] P. Muhly, Coordinates in operator algebra, (Book in preparation).

[9] A. L. T. Paterson, Groupoids, inverse semigroups, and their operator algebras, Progress in Mathematics, 170. Birkhäuser, Boston, 1999.

[10] J. Renault, A groupoid approach to $C^{*}$-algebras, Lecture Notes in Math. 793, Springer, Berlin, 1980.

[11] S. ZaIdman, Almost-periodic functions in abstract spaces, Research Notes in Math. 126. Pitman, Boston, 1985.

Manoscritto pervenuto in redazione il 20 novembre 2012. 
